\title{
POLÍTICAS E PROGRAMAS INSTITUCIONAIS DE APOIO A PERIÓDICOS CIENTÍFICOS EM UNIVERSIDADES PÚBLICAS PAULISTAS: CONTEXTUALIZAÇÃO E INDICADORES DE ATIVIDADE
}

\author{
INSTITUTIONAL POLICIES TO SUPPORT SCIENTIFIC JOURNALS IN \\ PUBLIC UNIVERSITIES OF SÃO PAULO STATE: CONTEXTS AND ACTIVITY \\ INDICATORS
}

\author{
Solange Alves Santana \\ Daisy Pires Noronha
}

Resumo: A pesquisa, de caráter exploratório descritivo, objetiva analisar políticas e programas institucionais de apoio a periódicos científicos implementados pelas universidades públicas estaduais paulistas, nomeadamente: Universidade de São Paulo (USP), Universidade Estadual Paulista "Júlio de Mesquita Filho" (UNESP) e Universidade Estadual de Campinas (UNICAMP), com vistas a identificar e mapear ações sistemáticas desenvolvidas e obter indicadores de atividade dos periódicos atendidos pelas instituições.

Palavras-chave: Comunicação científica. Periódicos científicos. Políticas institucionais.

Abstract: The purpose of this study was to analyze institutional policies implemented by public universities of São Paulo State - University of São Paulo (USP), São Paulo State University (UNESP) and University of Campinas (UNICAMP) - to support scientific journals. It also aims to identify systematic actions developed and to obtain activity indicators of the scientific journals attended by the institutions.

Keywords: Institutional policies. Scientific communication. Scientific journals.

\section{Introdução}

Os periódicos científicos ocupam espaço e função privilegiados na comunicação formal da pesquisa científica, sendo requeridos padrões de qualidade internacional tendo em vista que essa comunicação transcende fronteiras geográficas, temporais, culturais e linguísticas. A exigência desses padrões se reverte em aumento do custo de produção dos periódicos, exigindo maior aporte de recursos financeiros, em demanda de profissionalização de equipes editoriais, bem como na criação de sistemas de avalição de periódicos científicos que visam verificar o atendimento a critérios de qualidade (VALERIO, 1994; MIRANDA; PEREIRA, 1996; STUMPF, 1998; MEADOWS, 1999; BIOJONE, 2001; BARBALHO, 2009).

No cenário brasileiro, o aumento crescente do número de periódicos científicos observado nas últimas décadas, despertou a preocupação quanto à sustentabilidade financeira 
das publicações. Nascimento (2014) destaca o financiamento de periódicos científicos com recursos públicos, apontando como principais fontes o Programa Nacional de Apoio à Editoração e Publicação de Periódicos Científicos do Conselho Nacional de Desenvolvimento Científico e Tecnológico (CNPq), as fundações estaduais de apoio à pesquisa (FAPs) e universidades públicas. Essas fontes, em muitos casos, são combinadas a outras, tais como, cobrança de taxas de submissão e publicação, vendas de assinaturas e veiculação de anúncios.

Cumpre destacar que, no país, um número considerável de periódicos científicos é editado por universidades públicas e muitas delas desenvolvem algum tipo de política ou programa de apoio voltado às publicações científicas (MUELLER, 2011). Em estudo realizado, Rodrigues, Quartiero e Neuber (2015) identificaram 314 periódicos brasileiros indexados nas bases Web of Science e Scopus. Os resultados apontaram as universidades como o principal mantenedor de periódicos científicos identificados, com 147 títulos, correspondendo a $46,8 \%$ da amostra.

Segundo Bufrem (2003), a experiência universitária na edição de periódicos, que no Brasil data de 1960, evolui a partir de serviços gráficos, transmutando-se em editoras e, posteriormente, ao estabelecimento de políticas e programas de apoio aos periódicos científicos. Tais iniciativas, enquanto políticas institucionalizadas, estão diretamente associadas à necessidade de se fortalecer e aprimorar os periódicos científicos, enquanto linha de ação estratégica de apoio e divulgação da pesquisa científica brasileira.

As universidades públicas estaduais paulistas - Universidade de São Paulo (USP), Universidade Estadual Paulista "Júlio de Mesquita Filho" (UNESP) e Universidade Estadual de Campinas (UNICAMP) - ocupam lugar de destaque nesse cenário por serem responsáveis por um número considerável de periódicos indexados em bases de dados de relevância internacional. A título de exemplo, somente a USP possui 13 periódicos indexados na coleção Journal Citation Reports (JCR) da Thomson Reuters, conforme dados divulgados em 2016.

Diante do exposto, a pesquisa em andamento, realizada sob orientação da Profa. Dra. Daisy Pires Noronha, objetiva analisar políticas e programas institucionais de apoio a periódicos científicos implementados pelas universidades públicas estaduais paulistas, nomeadamente Universidade de São Paulo (USP), Universidade Estadual Paulista "Júlio de Mesquita Filho" (UNESP) e Universidade Estadual de Campinas (UNICAMP), com vistas à identificação e mapeamento das ações sistemáticas e levantamento de indicadores de atividade. 


\section{Políticas e programas de apoio a periódicos científicos nas universidades} públicas estaduais paulistas

Atualmente, as universidades públicas estaduais paulistas - USP, UNESP e UNICAMP - possuem políticas ou programas que visam garantir e apoiar a qualificação e a visibilidade das publicações periódicas científicas. Os dados disponibilizados em seus respectivos anuários estatísticos do ano de 2015 indicam que as três universidades contemplam um conjunto de 218 periódicos científicos em diferentes áreas do conhecimento (TABELA 1).

Tabela 1 - Periódicos atendidos pelos programas de apoio a periódicos científicos nas universidades públicas estaduais paulistas.

\begin{tabular}{lc}
\hline Universidade & Periódicos atendidos \\
\hline USP & $159^{(\mathrm{a})}$ \\
UNESP & $14^{(\mathrm{b})}$ \\
UNICAMP & $45^{(\mathrm{c})}$ \\
\hline
\end{tabular}

Fonte: (a) Anuário Estatístico Anual da Universidade São Paulo (USP) de $2015^{1}$, (b) Anuário Estatístico Anual Universidade Estadual Paulista "Júlio de Mesquita Filho" (UNESP) de $2015^{2}$, (c) Portal de Periódicos Eletrônicos da Universidade Estadual de Campinas (UNICAMP) ${ }^{3}$.

As políticas e programas visam não somente o apoio financeiro aos periódicos por meio de editais, mas também a qualificação e profissionalização das equipes, realização de eventos e a manutenção de portais direcionados aos periódicos (QUADRO 1), sendo desenvolvidas para sua operacionalização ações sistemáticas.

\footnotetext{
${ }^{1}$ Disponível em https://uspdigital.usp.br/anuario/AnuarioControle

${ }^{2}$ Disponível em https://ape.unesp.br/anuario/

${ }^{3}$ Disponível em http://periodicos.sbu.unicamp.br/wp/?page id $=113$
} 
VI Seminário de Pesquisa em Ciência da Informação do PPGCI 2017

Escola de Comunicações e Artes - Universidade de São Paulo

Quadro 1 - Programas implementados pela USP, UNESP e UNICAMP.

\begin{tabular}{|l|l|}
\hline Instituição & Política / Programa \\
\hline USP & $\begin{array}{l}\text { Mantem o Programa de Apoio às Publicações Científicas Periódicas da USP, } \\
\text { mantido pelo Sistema Integrado de Bibliotecas, desde 1986. Apoiado na filosofia do } \\
\text { acesso aberto, disponibiliza o Portal de Revistas USP, criado em 2008. }{ }^{\text {(a). }}\end{array}$ \\
\hline UNESP & $\begin{array}{l}\text { Mantem o Programa de Apoio às Publicações Científicas Periódicas da UNESP, } \\
\text { mantida pela Pró-Reitoria de Pesquisa. }\end{array}$ \\
\hline UNICAMP & $\begin{array}{l}\text { Mantem o Portal de Periódicos Eletrônicos Científicos (PPEC) da Universidade } \\
\text { Estadual de Campinas (UNICAMP), mantido pela Coordenadoria Geral da } \\
\text { Universidade (CGU) e pelo Sistema de Bibliotecas (SBU). }{ }^{\left({ }^{\prime}\right)}\end{array}$ \\
\hline
\end{tabular}

Fonte: (a) Sistema Integrado de Bibliotecas da Universidade de São Paulo (USP) ${ }^{4}$, (b) Pró-Reitoria de Pesquisa da Universidade Estadual Paulista "Júlio de Mesquita Filho" (UNESP) ${ }^{5}$, (c) Portal de Periódicos Eletrônicos da Universidade Estadual de Campinas (UNICAMP) ${ }^{6}$.

\section{Considerações parciais}

O desenvolvimento e implementação de políticas e programas de apoio aos periódicos científicos pelas universidades públicas estaduais paulistas se configura como uma ação estratégica ao fortalecimento e consolidação das publicações, uma vez que as agências de fomento atendem, cada vez mais, uma quantidade menor de títulos e nem sempre contemplam todas as áreas do conhecimento simultaneamente.

Tendo em vista os desafios impostos no cenário atual aos periódicos científicos, como gestão editorial profissionalizada, atendimento a critérios e padrões de qualidade internacionais em um contexto de competitividade, julga-se que a presente pesquisa poderá trazer contribuições à discussão acerca dos mecanismos de sustentabilidade financeira e da qualidade dos periódicos científicos brasileiros.

\section{Referências}

BARBALHO, C. R. S. Periódico científico: parâmetros para avaliação de qualidade. In: FERREIRA, S. M. S. P.; TARGINO M. G. (Org.). Preparação de revistas científicas: teoria e prática. São Paulo: Reichmann \& Autores Editores, 2005. p. 123-158.

BIOJONE, M. R. Os periódicos científicos na comunicação da ciência. São Paulo: Educ/Fapesp, 2003. 155 p.

BUFREM, L.S. Editoras universitárias no Brasil: uma crítica para a reformulação da prática. São Paulo/Curitiba: Edusp/Com-Arte/UFPR, 2003.

\footnotetext{
${ }^{4}$ Disponível em http://www.sibi.usp.br/bibliotecas/digitais-sistemicas/revista-usp/

${ }^{5}$ Disponível em http://unesp.br/portal\#!/prope/revistas-cientificas/revistas/

${ }^{6}$ Disponível em http://periodicos.sbu.unicamp.br/wp/?page id=113
} 
MEADOWS, A. J. A comunicação científica. Brasília: Briquet de Lemos, 1999. 268 p.

MIRANDA, D. B.; PEREIRA, M. N. F. A revista científica como veículo de comunicação: uma revisão de literatura. Ciência da Informação, v. 25, n. 3, p. 375-382, 1996.

MUELLER, S. P. M. O periódico científico. In: CAMPELLO, B. S.; CENDÓN, B. V.; KREMER J. M. (Org.). Fontes de informação para pesquisadores e profissionais. Belo Horizonte: UFMG, 2000. p. 73-96.

NASCIMENTO, C. C. Revistas científicas: a busca pela qualidade e indicadores bibliométricos. São Paulo, 2014. 126 f. Dissertação (Mestrado em Ciência da Informação) Programa de Pós-graduação em Ciência da Informação da Escola de Comunicações e Artes da Universidade de São Paulo, São Paulo, 2014.

RODRIGUES, R. S.; QUARTIERO, E.; NEUBERT, P. Periódicos científicos brasileiros indexados na Web of Science e Scopus: estrutura editorial e elementos básicos. Informação \& Sociedade, v.25, n.2, p. 117-138, 2015.

STUMPF, I. R. C. Reflexões sobre as revistas brasileiras. Intexto, v. 1, n. 3, p. 1-10, 1998.

VALERIO, P. M. Espelho da ciência: avaliação do Programa Setorial de Publicações em Ciência e Tecnologia da FINEP. Rio de Janeiro, Brasília: FINEP/IBICT, 1994.

\section{Sobre as autoras}

\section{Solange Alves Santana}

Mestranda em Ciência da Informação

Programa de Pós-Graduação em Ciência da Informação da Escola de Comunicação e Artes da Universidade de São Paulo (ECA-USP).

E-mail: sol@usp.br

\section{Daisy Pires Noronha}

Professora Sênior do Programa de Pós-Graduação em Ciência da Informação da Escola de Comunicação e Artes da Universidade de São Paulo (ECA-USP).

E-mail: daisynor@usp.br 